\title{
Insulin resistance as a non-invasive method for the assessment of fibrosis in patients with hepatitis $C$ : a comparative study of biochemical methods
}

\author{
M. Romera, R. Corpas and M. Romero Gómez \\ Unit for Clinical Management of Digestive Diseases. Hospital Universitario de Valme. Seville, Spain
}

\begin{abstract}
Introduction: insulin resistance (IR) promotes the progression of fibrosis and diminishes response to treatment in patients with hepatitis C. Recently, Sydney's index (includes IR) has been proposed as a non-invasive method for the prediction of fibrosis.

Objective: to assess the usefulness of Sydney's index for the prediction of advanced fibrosis (F3-F4) or absence of significant fibrosis (F0-F1) in patients with chronic hepatitis C.

Patients and methods: we included 131 patients suffering from chronic hepatitis $C$. Mean age was $40 \pm 11,78$ men and 53 women. Fibrosis stage was (F0-F1) 69 patients, F2: 40, and advanced (F3-F4) in 22 patients. We measured baseline AST, ALT, GGT, platelet, cholesterol, alcohol, and IR (HOMA - IR) levels. Sydney, Forns' and APRI indexes were calculated.

Results: the area under the curve for the diagnosis of absence of significant fibrosis in each method was: Sydney: 0.80, Forns: 0.71, APRI: 0.70; $\mathrm{p}=\mathrm{ns}$. Moreover, the diagnostic capacity of advanced fibrosis was: Sydney: 0.88, Forns: 0.83, APRI: 0.82; p $=$ ns. The predictive negative value of significant fibrosis was 74, 72 , and $67 \%$, respectively. Due to the presence of intermediate values, the indexes were not applicable to 36,44 and $43 \%$ of patients respectively.

Conclusions: the incorporation of insulin resistance among biochemical non-invasive methods slightly improves the yield of other indexes. Nevertheless, results are suboptimal, and more than one third of patients might not be correctly classified.
\end{abstract}

A part of this paper was selected for an oral presentation in the $64^{\text {th }}$ Annual Meeting of Sociedad Española de Patología Digestiva, which was awarded a prize to Best Clinical Presentation.

This study was supported by grants 43/03 and 102/04 from "Consejería de Salud”. CTS-532 Consejería de Innovación. Junta de Andalucía. Spain.

Recibido: 22-08-05.

Aceptado: 10-11-05.

Correspondencia: Manuel Romero Gómez. Unidad de Gestión Clínica de Enfermedades Digestivas, $2^{\mathrm{a}}$ planta. Hospital Universitario de Valme. Ctra. de Cádiz, s/n. 41014 Sevilla. Fax: 955015 899. e-mail: mromerog@supercable.es
Key words: Alanin-aminotransferase. Aspartate-aminotransferase. Transaminases. Platelets. Cholesterol. Non-invasive methods. Hepatitis C. Fibrosis. Gamma-glutamyl-transpeptidase.

Romera M, Corpas R, Romero Gómez M. Insulin resistance as a non-invasive method for the assessment of fibrosis in patients with hepatitis $C$ : a comparative study of biochemical methods. Rev Esp Enferm Dig 2006; 98: 161-169.

\section{ABBREVIATIONS}

IR: insulin resistance.

HOMA: homeostasis model assessment.

APRI: ast platelet ratio index.

GGT: gammaglutamyl-transpeptidase.

ALT: alanin-aminotransferase.

AST: aspatartate-amino-transferase.

AUC: area under curve.

PPV: positive predictive value.

\section{INTRODUCTION}

Hepatitis $\mathrm{C}$ is a worldwide disease that affects $1-3 \%$ of the population, depending on geographical areas. In Spain its prevalence has been estimated around 2\% (1). The knowledge of fibrosis stage is very useful in the management of patients with hepatitis $\mathrm{C}$, as it allows to value need of treatment, and to establish a prognosis. Although liver percutaneous biopsy continues to be the only diagnostic method that delimits fibrosis, new non-invasive models have been designed, based both in biochemical and imaging technologies. Biochemical models can be classified in four types: a) models using parameters obtained in clinical practice [Forns': (platelets, GGT, cholesterol and age) (2) and APRI index: (platelets and AST)] (3); b) models including biochemical parameters not measured in clinical 
practice [Fibrotest (alpha 2 macroglobulin, apolipoprotein A1, haptoglobin, GGT, and total bilirubin)] (4); c) indexes including markers of fibrogenesis such as peptide-procollagen III, hyaluronic acid, and YKL-40 (5,6); and d) models using parameters obtained by means of proteomic approaches for the study of glycoproteins (7). Hepatitis C promotes the development of insulin resistance, probably because of a direct effect of the virus on the intracellular insulin signaling pathway (8). Besides, the role of insulin resistance has been stated in the progression of disease (9). In euglycemic patients the calculation of HOMA's index allows to quantify insulin resistance. A correlation between fibrosis and insulin resistance has been demonstrated (10). A group from the University of Sydney (11) proposed a non-invasive model for fibrosis prediction that included insulin resistance together with age, AST, cholesterol, and alcohol use. This Sydney model has demonstrated a high capacity for fibrosis prediction in patients with hepatitis $\mathrm{C}$. In view of the enormous interest that non-invasive methods have for standard clinical practice, we designed the present study to validate the usefulness of Sydney's index in a group of patients with hepatitis $\mathrm{C}$ in our Unit.

\section{PATIENTS AND METHODS}

We reviewed the medical records of 131 patients with chronic hepatitis $\mathrm{C}$ submitted to percutaneous hepatic biopsy between 1999 and 2003 in the Unit of Hepatology of Valme University Hospital, of which there were available data regarding platelets, AST, cholesterol, age, gamma-glutamyl-transpeptidase (GGT), HOMA-IR, and information on the degree of alcohol consumption at the time of liver biopsy. They all had anti-HCV and positive HCV RNA. No patient was an AgHBs or anti-HIV carrier, or had any previous antiviral treatment. It was a series of 78 men and 53 women with a mean age of $40 \pm 11$ (22-67). Genotype 1: 56 (42.7\%); genotype 2/3: 25 (19.1\%), and an unknown genotype in $50(38.2 \%)$. The stage of fibrosis was evaluated between F0 and F4 using Scheuer's classification (12). All biopsies were evaluated by the same pathologist, who selected those with an optimal size. The average number of portal tracts was $10 \pm 2$. Patients were classified according to fibrosis into three groups: mild fibrosis (F0-F1), advanced fibrosis (F3-F4), and significant fibrosis (F2-F3F4). We calculated insulin resistance using the HOMA $($ Homeostasis Model Assessment test $)=$ insulin $(\mathrm{mU} / \mathrm{ml}) *$ glucemia $(\mathrm{mMol} / \mathrm{L}) / 22.5$. All biochemical parameters, histologic information, HOMA-IR, and alcohol consumption for the 131-patient sample are listed in Table I. We reviewed the medical records and gathered information about GGT, age, cholesterol, and platelets to calculate Forns's index according to the formula: [7.811-3.131 * In $\left(\mathrm{n}^{\circ}\right.$ platelets) $+0.781 * \ln (\mathrm{GGT})+3.467 * \ln ($ age $)-0.014 *$ (cholesterol)]. Patients were classified according to the cutoffs indicated by Forns et al. into three different groups:
Table I. Histological, biochemical, hematological, and environmental parameters of patients included

\begin{tabular}{ll}
\hline Fibrosis (Scheuer) & \\
Fibrosis F0 & $10(7.8 \%)$ \\
Fibrosis F1 & $59(45 \%)$ \\
Fibrosis F2 & $40(30.5 \%)$ \\
Fibrosis F3 & $8(6.1 \%)$ \\
Fibrosis F4 & $14(16.7 \%)$ \\
\hline Platelets & $198,000(56,000)$ \\
\hline Cholesterol & $170(38)$ \\
\hline AST & $78.6(66)$ \\
\hline ALT & $130(149)$ \\
\hline GGT & $74(94)$ \\
\hline Alcohol & 76 patients $(59 \%)$ \\
Abstemious or < $10 \mathrm{~g} / \mathrm{d}$ consumers & 20 patients $(16 \%)$ \\
$10-40 \mathrm{~g} / \mathrm{d}$ consumers & 32 patients $(25 \%)$ \\
$>40 \mathrm{~g} / \mathrm{d}$ consumers & 3 patients \\
Unknown &
\end{tabular}

$<4.2$ points [ $\mathrm{n}=46(41.4 \%)$ ], between 4.2 and 6.9 points $[(n=49(41.1 \%)]$, and $>6.9$ points $[n=16(14.41 \%)]$. The APRI was calculated as the number of times AST was greater than normal/number of platelets: $\left[n^{\circ}\right.$ times AST > normal value/platelets*10* 100]. AST normal value was $37 \mathrm{U} / \mathrm{L}$, and platelets normal value was 142.000 platelets/microliter for our laboratory. Patients were also classified according to the cutoffs suggested by Wai et al.: APRI $<0.50[\mathrm{n}=37(33.1 \%)]$, between 0.5 and $1.5[(\mathrm{n}=$ $48 ; 42.8 \%)]$, and APRI $>1.5$ [ $(\mathrm{n}=27 ;(24.1 \%)]$. The Sydney index was calculated using the formula [e*/1 + and $*]$, where '*' is $[-10.929+(1.827 * \operatorname{LnAST})+(0.081 *$ age $)$ $+(0.768 *$ alcohol graduate of $0-2)+(0.385 *$ HOMA-IR $)$ - $(0.447 *$ cholesterol in mmol) $)$. To calculate the HOMA index, insulinemia was determined by means of ECLIA in samples frozen to $-80{ }^{\circ} \mathrm{C}$ after collection at the time of liver biopsy. Patients were classified into three groups according to the threshold indicated by Sud et al.: Sydney $<0.2[\mathrm{n}=$ $54(45 \%)$ ], between 0.2 and 0.9 [n= $51(42.5 \%)]$ and Sydney $>0.9[\mathrm{n}=15(12.5 \%)]$.

\section{Statistical analysis}

We calculated Forns', APRI, and Sydney indexes for each patient. We analyzed the ability to predict mild fibrosis (F0-F1) and to confirm advanced fibrosis (F3-F4) by means of the ROC curve. In addition, using the Epidat 3.0 (Xunta of Galicia, Santiago de Compostela, Spain) software program we calculated the sensitivity, specificity, positive predictive value, and negative predictive value, as well as the $95 \%$ confidence interval, of every parameter for each cutoff value in the exclusion of significant fibrosis and the detection of advanced fibrosis. Using the SPSS 12.0 (SPSS, Chicago, IL) program we performed a 
multivariate study to analyze the role of every single variable in the prediction of mild fibrosis. We presented all data as mean \pm standard deviation. We considered significance for $\mathrm{p}<0.05$.

\section{RESULTS}

We detected a correlation between each of the indexes and fibrosis: Forns' index $(\mathrm{r}=0.46 ; \mathrm{p}<0.001)$, APRI index $(r=0.48 ; p<0.001)$, and Sydney index $(r=0.59 ; p<0.001)$.

\section{Usefulness of the three methods in the prediction of absence of significant fibrosis (F0-F1)}

The analysis by means of the ROC curve demonstrated that the area under the curve of the Sydney index regarding diagnostic capacity for absence of significant fibrosis is higher than that of the other two models (Sydney's index AUC $=0.80$ ), whereas Forns' and APRI models had a similar diagnostic capacity with an AUC of 0.71 and 0.70, respectively (Fig. 1). The model from Sydney reached a negative predictive value for significant fibrosis of $74 \%$, so in 54 patients we detected a Sydney index lower than 0.2 and, out of them, 40 (74\%) had F0-F1 fibrosis. Therefore, Forns' model reached a negative predictive value for significant fibrosis of $72 \%$, since out of 46 patients with a Forns' index $<4.2$, 33 cases $(72 \%)$ had F0-F1 fibrosis, and the APRI model reached a negative predictive value for significant fibrosis of $67 \%$, so that of 37 patients with an APRI index $<0.5,25$ had non-significant fibrosis.

\section{Usefulness of the three methods in the prediction of advanced fibrosis (F3-F4)}

The area under the ROC curve of the diagnosis of advanced fibrosis was 0.88 for the Sydney index, and 0.83 and 0.82 for Forns' and APRI indices, respectively (Fig. 2). Patients with a result above 0.9 in the Sydney index showed a positive predictive value of advanced fibrosis of $73 \%$, since 10 out of 15 patients with a Sydney result $>0.9$ had advanced fibrosis (F3-F4); a Forns' index $>6.9$ demonstrated a positive predictive value of $75.2 \%$, since out of 16 patients with a Forns' score $>6.9,12$ cases were showing fibrosis $(\mathrm{F} 3-\mathrm{F} 4)$. Patients who had an APRI $>1.5$ showed a positive predictive value of advanced fibrosis of only $55 \%$, since of 27 patients with an APRI index $>1.5$, only 15 had fibrosis (F3-F4).

\section{Relationship between fibrosis and the included variables}

Univariate analysis:

To analyze the weight of every variable we performed a linear regression between fibrosis and each of the studied parameters. We found a correlation between fibrosis and insulin resistance $(r=0.37 ; p<0.001)$, age $(r=0.43$; $\mathrm{p}<0.001)$, alcohol use $(\mathrm{r}=0.22 ; \mathrm{p}=0.013)$, number of platelets $(\mathrm{r}=-0.45 ; \mathrm{p}<0.001)$, AST $(\mathrm{r}=0.35 ; \mathrm{p}<0.001)$, and GGT ( $\mathrm{r}=0.33$; $\mathrm{p}<0.001)$; however, we found no correlation with cholesterol levels $(r=0.06 ; p=0.37)$.
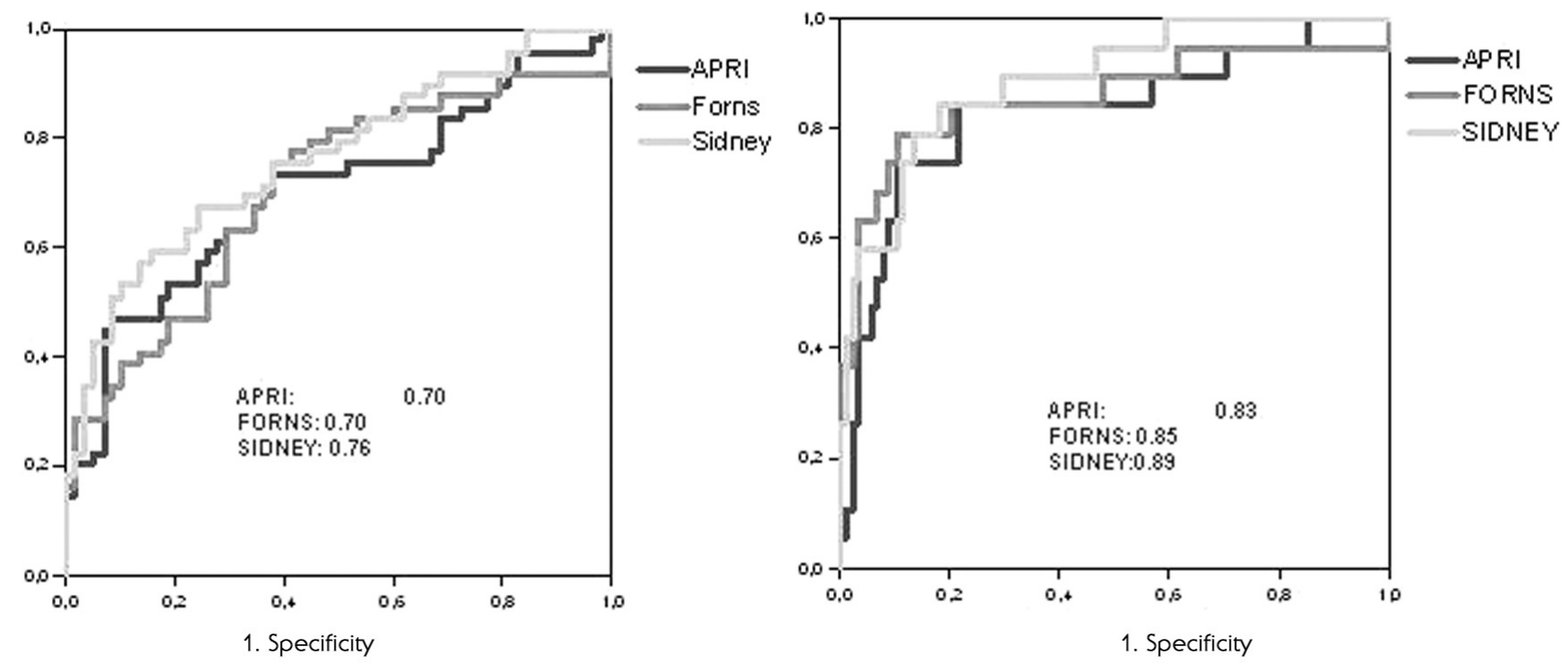

Fig 1.- Analyses of the ability to diagnose non-significant fibrosis (A) and advanced fibrosis (B) using the ROC curve.

Análisis de la capacidad de predicción de ausencia de fibrosis significativa y fibrosis avanzada usando el área bajo la curva ROC. 
Table II. Multivariate analyses of a multiple linear regression to analyze the influence of each variable on liver fibrosis. We found that AST, platelets, age, and HOMA - IR were independent variables associated with fibrosis. GGT and cholesterol levels did not relate significantly

\begin{tabular}{lccc}
\hline & $\begin{array}{c}\text { Coefficient } \\
\text { Beta }\end{array}$ & $T$ & Sig. \\
\hline (Constant) & 0.233 & 0.862 & 0.391 \\
AST & 0.055 & 0.637 & 0.006 \\
GGT & -0.262 & -3.147 & 0.526 \\
Platelets & 0.231 & 2.791 & 0.002 \\
AGE & 0.193 & 2.275 & 0.025 \\
HOMAIR & & & \\
\hline
\end{tabular}

Dependent variable: fibrosis.

Multivariate analysis

A multiple linear regression model was configured $\left(\mathrm{r}^{2}\right.$ $=0.36)$, in which age $(\mathrm{p}=0.006)$, platelet number $(\mathrm{p}=$ $0.001)$, AST $(p=0.006)$, and HOMA-IR $(p=0.02)$ were defined as independent variables for fibrosis prediction (Table II).

\section{DISCUSSION}

Determining the Sydney index in patients with hepatitis C slightly improves the yield of methods such as Forns' or APRI, although this improvement is very small and stays far from clinical and statistical significance. The main problems of non- invasive methods for fibrosis prediction in hepatitis $\mathrm{C}$ remain unsolved after the incorporation of insulin resistance in the model, and despite its narrow association with fibrosis. The most important limitations detected in the validation studies of the various non-invasive methods for fibrosis prediction in hepatitis $\mathrm{C}$ are: a) on the one hand, between a third and half of patients have intermediate values in which we cannot predict fibrosis; b) the negative predictive value of significant fibrosis is clearly below that reported by authors in their validation series, and stay far from the requirable quality standards (> 85\%). Forns' index conveyed a NPV of $96 \%$ that decreased to $71.4 \%$ in a subsequent group. Also, APRI fell from 86 to $72.4 \%$ (13) and in the current study, the Sydney index had NPV for significant fibrosis of $70 \%$, in contrast to the $93 \%$ reported by the authors. These discrepancies could be explained by the different statistical power and the prior prevalence of fibrosis in the reported studies. Besides, most methods separate well advanced fibrosis (F3-F4) from stage-2 fibrosis. However, the ability to separate F1 from F2 is still subopti$\mathrm{mal}$; and c) in the multivariate analyses leading to the design of the formula to calculate these indexes variables such as cholesterol are still included, which in our cohort are not related to fibrosis. For all this, though this is an area of great interest for the practice of medicine, these limitations do not seem to be corrected by the incorporation of markers of fibrogenesis (14) or markers related to fibrosis such as insulin resistance.

Fibrosis staging in patients diagnosed with hepatitis $\mathrm{C}$ is clinically relevant, as it allows us to determine the prognosis, as well as the need to receive treatment. Nowadays, percutaneous hepatic biopsy with echographic control continues to be the gold standard to delimit fibrosis stages. Liver biopsy has a number of limitations, among which intra- (15) and inter-observer (16) variability must be emphasized -the sample error that can miss a cirrhosis diagnosis in 15 to $20 \%$ of cases (17). In a group of 124 patients with hepatitis $\mathrm{C}$ submitted to laparoscopy with biopsy collection from both hepatic lobes, it was verified that in one third of cases a difference in stage between biopsies was detected, and that in $15 \%$ of cases liver cirrhosis was confirmed in only one lobe (18).

Recently, it has been stated that sample size is very important for fibrosis staging reproducibility. In small biopsies, the pathologist tends to underestimate fibrosis, whereas in biopsies greater than $25 \mathrm{~mm}$ the valuation of fibrosis is more reproducible $(19,20)$. Nevertheless, this increase in sample size also increases the risk of complications. Anyway the risk of suffering a motor vehicle accident on the way to hospital is thought to be higher than mortality in relation to liver biopsy (http: //www.dgt.es/).

Diagnostic possibilities when using these non-invasive predictive models of fibrosis improve with the use of a combination of several models. Considering patients with genotype 1, using Forns' score together with APRI allows to improve the negative predictive value for significant fibrosis up to $82 \%$ (13). This figure is supported by a better result obtained using the Fibrotest together with elastography (21). Due to the limitations of biochemical methods, new image techniques are being developed for the estimation of hepatic fibrosis. The use of transient elastography is a promising method that has been validated in external groups (22). Moreover, the optical analysis of computerized tomography liver images from patients with hepatitis $\mathrm{C}$ allows to assess the stage of liver fibrosis (23).

The ideal diagnostic method for liver fibrosis must be simple, cheap, safe, and non-invasive. In a validation study including 1,252 patients, a PPV of $58.2 \%$ for advanced fibrosis was reported using Forns' method, versus $75.2 \%$ as obtained in the current study. Similarly, in the current study, as reported by Iacobellis et al. (24), PPV was 55\% for advanced fibrosis using the APRI index. These figures would confirm a drop in the performance of these tests in validation studies.

In conclusion, the development of simple models including biochemical parameters used in regular clinical practice permits to predict liver fibrosis in patients with hepatitis C. Nevertheless, further improvement of these methods is still needed, as results are suboptimal and fibrosis assessment has a deep impact on the management of patients with chronic hepatitis C. 


\section{REFERENCES}

1. Eiras A, Sauleda S, Planelles D, et al. HCV screening in blood donations using RT-PCR in mini-pool: the experience in Spain after routine use for 2 years. Transfusion 2003; 43: 713-20.

2. Forns X, Ampurdanes S, Llovet JM, et al. Identification of chronic hepatitis $\mathrm{C}$ patients without hepatic fibrosis by a simple predictive model. Hepatology 2002; 36: 986-92.

3. Wai CT, Greenson JK, Fontana RJ, Kalbfleisch JD, Marrero JA, Conjeevaram HS, et al. A simple noninvasive index can predict both significant fibrosis and cirrhosis in patients with chronic hepatitis C. Hepatology 2003; 38: 518-26.

4. Imbert-Bismut F, Ratziu V, Pieroni L, Charlotte F, Benhamou Y, Poynard T. Biochemical markers can of liver fibrosis in patients with hepatitis C virus infection: a prospective study. Lancet 2001; 357: 1069-75.

5. Saitou Y, Shiraki K, Yamanaka Y, et al. Noninvasive estimation of liver fibrosis and response to interferon therapy by a serum fibrogenesis marker, YKL-40, in patients with $\mathrm{HCV}$-associated liver disease. World J Gastroenterol 2005; 11: 476-81.

6. Patel K, Gordon SC, Jacobson I, et al. Evaluation of a panel of noninvasive serum markers to differentiate mild from moderate-to-advanced liver fibrosis in chronic hepatitis C patients. J Hepatol 2004; 41: 935-42.

7. Callewaert N, Van Vlierberghe H, Van Hecke A, Laroy W, Delanghe $\mathrm{J}$, Contreras R. Noninvasive diagnosis of liver cirrhosis using DNA sequencer-based total serum protein glycomics. Nat Med 2004; 10 : 429-34.

8. Kawaguchi T, Yoshida T, Harada M, et al. Hepatitis $\mathrm{C}$ virus down-regulates insulin receptor substrates 1 and 2 through up-regulation of suppressor of cytokine signaling 3. Am J Pathol 2004; 165: 1499-508.

9. Fartoux L, Poujol-Robert A, Guechot J, Wendum D, Poupon R, Serfaty $\mathrm{L}$. Insulin resistance is a cause of steatosis and fibrosis progression in chronic hepatitis C. Gut 2005; 54: 1003-8.

10. Romero-Gomez M, Del Mar Viloria M, Andrade RJ, et al. Insulin resistance impairs sustained response rate to peginterferon plus ribavirin in chronic hepatitis C patients. Gastroenterology 2005; 128: 63641.

11. Sud A, Hui JM, Farrell GC, et al. Improved prediction of fibrosis in chronic hepatitis $\mathrm{C}$ using measures of insulin resistance in a probability index. Hepatology 2004; 39: 1239-47.

12. Romero-Gómez M, Ramírez Martín del Campo M, Otero MA, Vallejo M, Corpas R, Castellano-Megias VM. Comparative study of two models that use biochemical parameters for the non-invasive diagnosis of fibrosis in patients with hepatitis C. Med Clin (Barc) 2005; 124: 761-4.

13. Rosenberg WM, Voelker M, Thiel R, et al. Serum markers detect the presence of liver fibrosis: a cohort study. Gastroenterology 2004; 127: $1704-13$

14. Goldin RD, Goldin JG, Burt AD, Dhillon PA, Hubscher S, Wyatt J, Patel N. Intra-observer and inter-observer variation in the histopathological assessment of chronic viral hepatitis. J Hepatol 1996; 25: 64954.

15. Westin J, Lagging LM, Wejstal R, Norkrans G, Dhillon AP. Interobserver study of liver histopathology using the Ishak score in patients with chronic hepatitis C Virus infection. Liver 1999; 19: 183-7.

16. Soloway RD, Baggenstoss AH, Schoenfield LJ, Summerskill WH Observer error and sampling variability tested in evaluation of hepatitis and cirrhosis by liver biopsy. Am J Dig Dis 1971; 16: 1082-6.

17. Regev A, Berho M, Jeffers LJ, et al. Sampling error and intraobserver variation in liver biopsy in patients with chronic $\mathrm{HCV}$ infection. Am J Gastroenterol 2002; 97: 2614-8.

18. Bedossa P, Dargere D, Paradis V. Sampling variability of liver fibrosis in chronic hepatitis C. Hepatology 2003; 38: 1449-57.

19. Guido M, Rugge M. Liver biopsy sampling in chronic viral hepatitis. Sem Liver Disease 2004; 24: 89-97.

20. Giannini E, Risso D, Botta F, Chiarbonello B, Fasoli A. Validity and clinical utility of the aspartate aminotransferase - alanine aminotransferase ratio in assessing disease severity and prognosis in patients with hepatitis C Virus - related chronic liver disease. Arch Intern Med 2003; 163: 218-24.

21. Castera L, Vergniol J, Foucher J, et al. Prospective comparison of transient elastography, Fibrotest, APRI, and liver biopsy for the assessment of fibrosis in chronic hepatitis C. Gastroenterology 2005; 128: 343-50.

22. Ziol M, Handra-Luca A, Kettaneh A, Christidis C, et al. Noninvasive assessment of liver fibrosis by measurement of stiffness in patients with chronic hepatitis C. Hepatology 2005; 41: 48-54.

23. Romero-Gómez M, Gómez-González E, Vera-Valencia M, et al. Fibrosis assessment in hepatitis $\mathrm{C}$ by computer-performed optical analysis of CT of the liver: A novel non-invasive and useful method. 40th Annual meeting of the European Association for the study of the liver. Paris April 13-17, 2005. p. 599A.

24. Iacobellis A, Mangia A, Leandro G, Clemente R, Festa V, Attino V, et al. External validation of biochemical indices for noninvasive evaluation of liver fibrosis in HCV chronic hepatitis. Am J Gastroenterol 2005; 100: 868-73 